\title{
Review of: "Programmable microbial ink for 3D printing of living materials produced from genetically engineered protein nanofibers"
}

\author{
Xuan Mu
}

Potential competing interests: The author(s) declared that no potential competing interests exist.

Biological systems exhibit unique material and structural features, such as ambient and aqueous conditions, proteinaceous building blocks, complicated feedback systems, and hierarchical molecular assembly, which remain largely inaccessible to artificial systems and manufacturing. Using bioinspired principles has been of intense interest to develop bioinks and 3D bioprinting mechanisms.(1) For example, 3D bioprinting has realized hierarchical organization,(2) monolithic protein inks with structural integrity,(3, $4)$ and integrated "living" biofunctions, $(5,6)$ underlying the promise of 3D prints in a wide range of biomedical applications.

The paper "programmable microbial ink for 3D printing of living materials produced from genetically engineered protein nanofibers" describes another interesting bioinspired 3D printing method with microbial inks.(7)

First, the microbial ink is made entirely by E. coli, containing both living cells and nanofibrous biofilms, eliminating the need to incorporate extra, synthetic materials.(8) The viscoelastic properties of the microbial ink can be manipulated by genetic means for optimizing extrusion-based printability. For example, the "knob" and "hole" domains, analogous to the assembly domains of fibrin, have been added to biofilm nanofibers for promoting assembly. As a result, the microbial ink exhibits increased viscosity, shear modulus, storage modulus, and yield stress by several folds, as well as enhanced printability, such as the over $16 \mathrm{~mm}$ length of a printed overhanging filament. The authors also successfully printed two 10layered structures and a 21-layer solid cone with a printing filament of around $0.5 \mathrm{~mm}$ diameter. Yet, a rheological thixotropic test, missed here, may be necessary to quantitatively characterize the recovery of the yield stress of the ink after extrusion.

Second, the microbes in the inks remain alive, thus enabling the adoption of a wide range of genetic engineering approaches for rending 3D prints with various biofunctions, including the production of therapeutic molecules, toxic chemical sequester, and regulated cell growth. In particular, ITPG, a chemical inducer, has been used to induce the genetically engineered E. coli, encapsulated in a 2D print, to secrete azurin, an anticancer drug. Also, a BPA-binding peptide has been genetically engineered to add on the nanofibers, thus allowing a 2D print to sequester BPA from the surrounding environment. Furthermore, 
MazF, an endoribonuclease toxin, has been added to the genome of E. coli for regulating cellular growth and most likely inducing cell death. These exceptional examples would be promising for other clinical applications, such as wound dressing, drug delivery, and health monitoring.

\section{References:}

1. Y. S. Zhang et al., 3D extrusion bioprinting. Nature Reviews Methods Primers 1, 75 (2021).

2. M. R. Sommer, M. Schaffner, D. Carnelli, A. R. Studart, 3D printing of hierarchical silk fibroin structures. ACS Appl. Mater. Interfaces 8, 34677-34685 (2016).

3. X. Mu et al., 3D Printing of Silk Protein Structures by Aqueous Solvent-Directed Molecular Assembly. Macromol. Biosci. 20, 1900191 (2020).

4. X. Mu, V. Fitzpatrick, D. L. Kaplan, From Silk Spinning to 3D Printing: Polymer Manufacturing using Directed Hierarchical Molecular Assembly. Adv. Healthcare Mater. 9, 1901552 (2020).

5. M. Schaffner, P. A. Rühs, F. Coulter, S. Kilcher, A. R. Studart, 3D printing of bacteria into functional complex materials. Sci. Adv. 3, eaao6804 (2017).

6. X. Liu et al., 3D printing of living responsive materials and devices. Adv. Mater. 30, 1704821 (2018).

7. A. M. Duraj-Thatte et al., Programmable microbial ink for 3D printing of living materials produced from genetically engineered protein nanofibers. Nat. Commun. 12, 6600 (2021).

8. A. M. Duraj-Thatte et al., Genetically Programmable Self-Regenerating Bacterial Hydrogels. Adv. Mater. 31, 1901826 (2019). 